12. Delgado J, Laurini M. Lesiones traumáticas del diafragma. Monografía del postgrado en cirugía general. Facultad de Medicina. Hospital Maciel Montevideo, Uruguay. 2001.

13. Vázquez LA, Rodríguez Y, Pila R, Pérez M. Hernia diafragmática traumática: "la gran imitadora". Archivo Médico de Camagüey 2006; 10 (5) ISSN 1025-0255. Accedido el 11 de enero de 2007 en http:// www.amc.sld.cu/amc/2006/v10n5-2006/2079.pdf

14. Patitó J, Lossetti O, Trezza F, Guzmán C, Stingo N. Tratado de medicina legal y elementos de patología forense. Primera edición. Argentina: Editorial Quórum. 2003. Pág. 296-298

15. Gisbert JA. Medicina Legal y Toxicología. Quinta edición. España: Editorial Masson. 2001. Pág. 206
16. Knight B. Medicina forense de Simpson. Segunda edición. México: Editorial El Manual Moderno. 1999. Pág. 135.

17. Kvitko LA. Factores de la causalidad médico legal. Cátedra de Medicina Legal y Deontología Médica. Facultad de Medicina. Universidad de Buenos Aires. Accesado el 17 de febrero de 2007 de http://www.mednet.org.uy/dml/bibliografia/exterior/lak-200309.htm

\title{
Tuberculosis hepática primaria como causa de fiebre de origen desconocido
}

\author{
Manuel Antonio Villalobos-Zúñiga, Júvel Quintanilla-Gallo, Charles Gourzong-Taylor.
}

\section{Resumen}

Se presenta el caso de un paciente de 29 años de edad, quien se presentó con un cuadro de fiebre, sudoración nocturna y pérdida de peso, el que inicialmente fue catalogado como dengue clásico, pero que al persistir, fue abordado como fiebre de origen desconocido. Debido a que las pruebas de laboratorio y gabinete iniciales no fueron concluyentes, fue necesario realizar una laparotomía exploratoria para obtener material histológico en el que se demostraron granulomas caseosos en el hígado y formas similares a micobacterias con las tinciones especiales. Con el tratamiento antifímico, se observó una respuesta clínica favorable, por lo que se concluye que el cuadro corresponde a una probable TB hepática.

Descriptores: tuberculosis hepática, fiebre de origen desconocido, granulomas caseosos.

Key Words: hepatic tuberculosis, fever of unknown origin, granulomas caseousus.

Recibido: 30 de mayo de 2007

Aceptado: 6 de marzo de 2007

La mayoría de los cuadros febriles agudos o subagudos corresponden a infecciones virales o bacterianas, identificables de forma sencilla con una buena historia clínica, un examen físico completo y algunos exámenes de laboratorio y gabinete rutinarios. Cuando el cuadro persiste con fiebre de $38.3^{\circ} \mathrm{C}$ o más, confirmada en varias tomas, y por al menos 3 semanas, sin diagnóstico a pesar de las indagaciones clínicas iniciales durante una semana de estudio exhaustivo intrahospitalario, la entidad clínica se denomina

ISSN 0001-6002/2007/49/4/222-225

Acta Médica Costarricense, $\odot 2007$

Colegio de Médicos y Cirujanos fiebre de origen desconocido (FOD). La nueva definición también abarca la posibilidad de estudiar al paciente de forma ambulatoria en un mínimo de 3 consultas. ${ }^{1,2}$

Para estudiar un caso de FOD como el que se expone, debe aplicarse una serie de estudios clínicos especializados, orientados a descartar enfermedades infecciosas poco comunes en frecuencia y localización, por ejemplo, tuberculosis (TB) ó micosis, enfermedades neoplásicas como el linfoma y enfermedades de estirpe reumatológico. Lo anterior se deduce de las series de casos de FOD expuestas en la bibliografía, ${ }^{3}$ donde se demuestran las causas más frecuentes de esta entidad.

\section{Caso clínico}

Paciente masculino de 29 años de edad, nicaragüense, vecino de San José, peón de construcción. Consumidor de 3 cigarrillos de tabaco diarios durante 19 años, alcohol de forma ocasional y marihuana en el pasado y en poca cantidad. Sin antecedentes patológicos conocidos. Ingreso a emergencias médicas en diciembre de 2005, por fiebre continua de 3 días de evolución, cefalea frontal, mialgias y malestar general, síntomas inespecíficos que unidos al antecedente de haber estado recientemente en Puntarenas y a la presencia de plaquetopenia en los exámenes iniciales, merecieron el diagnóstico de dengue clásico. Así fue egresado, pero la fiebre persistió por al menos 21 días, por lo cual reconsulta en enero de 2006 (4 semanas después), con los mismos síntomas, pero con un patrón de fiebre con sudoración nocturna y pérdida de peso no cuantificada, por lo que se interna bajo el diagnóstico de FOD clásica.

El examen físico reveló un paciente adelgazado, agudamente enfermo, febril $\left(40^{\circ} \mathrm{C}\right.$ en promedio), con 2 adenopatías cervicales móviles y no dolorosas, y un soplo pan-sistólico grado II, sin otros hallazgos de importancia. Se 
realizaron los siguientes estudios, todos dentro de límites normales: hemograma, función renal, función hepática, examen general de orina, urocultivo, hemocultivo, VDRL, serología por virus de inmunodeficiencia humana (VIH), gota gruesa, serología por dengue, toxoplasmosis, EpsteinBarr, virus de hepatitis B y C, antígenos febriles (brucela, salmonela y Shigella), marcadores tumorales, función tiroidea, radiografía de tórax y de senos paranasales, TAC y US de abdomen, ecocardiograma, análisis de líquido cefalorraquídeo, esputo por bacilo de Koch (BK), factor antinúcleo, factor reumatoide, anti estreptolisina $\mathrm{O}$, anticuerpos anticardiolipina, inmunoglobulinas $\mathrm{y}$ complemento, gastroscopía, colon por enema y médula ósea. Una broncoscopía no encontró lesiones endobronquiales y el lavado bronquiolo alveolar fue negativo por bacterias, micobacterias y hongos.

Los hallazgos anormales fueron pocos e inespecíficos, eritrosedimentación elevada (VES: $75 \mathrm{~mm} / \mathrm{h}$ ), proteína $\mathrm{C}$ reactiva elevada (PCR: $16 \mathrm{mg} / \mathrm{dl}$ ), fibrinógeno: $755 \mathrm{mg} / \mathrm{dl}$, deshidrogenasa láctica: $338 \mathrm{U} / \mathrm{L}$ y tuberculina positiva con una pápula de $10 \mathrm{~mm}$ de induración.

Con esta información se descartó una gran cantidad de entidades causales de FOD, pero aún el diagnóstico no fue claro; por esta razón se realizó una laparotomía exploratoria, 6 semanas después del inicio de la fiebre, para obtener histología hepática, y macroscópicamente se reportó: "hígado con lesiones blanquecinas, redondeadas, granulomatosas en ambos lóbulos hepáticos. Adenopatías mesentéricas y paraaórticas. No hay masas en yeyuno e ileon. Apéndice sin lesiones. Bazo grado I sin lesiones focales. Escaso líquido peritoneal”.

La biopsia de los ganglios fue descrita sin cambios patológicos, el líquido peritoneal con cambios reactivos y la biopsia hepática con granulomas caseificantes, y formas sugestivas de bacilos alcohol ácido resistentes (Figura 1). No se observaron hongos, por lo que se estableció el diagnóstico de tuberculosis hepática. Se inició tratamiento antifímico tetra-asociado con una respuesta clínica favorable, mejoría del estado general, desaparición de la fiebre y ganancia de peso a los 2 meses de tratamiento.

\section{Discusión}

La definición de FOD incluye fiebre confirmada de $\geq$ $38.3^{\circ} \mathrm{C}$, persistiendo sin diagnóstico por al menos 3 semanas, a pesar de 1 semana de estudio diagnóstico en un hospital. ${ }^{1,2}$ La anterior descripción corresponde a la FOD clásica, a la cual se le unen 3 nuevas categorías, según la epidemiología actual mundial (propuestas por Durack y Steet): ${ }^{2}$ FOD nosocomial si no presentaba fiebre 3 días previos a su admisión hospitalaria, FOD en el paciente inmunodeficiente principalmente neutropénicos y FOD relacionada al VIH. Otros han incluido FOD en el paciente con cáncer, en el niño $\mathrm{o}$ en el anciano, ${ }^{3}$ pero en la práctica diaria su uso es infrecuente. Tales clasificaciones permiten proponer diagnósticos etiológicos, según su frecuencia en las distintas categorías.

Las 5 principales categorías etiológicas de FOD son, en orden de frecuencia: enfermedades infecciosas, neoplasias, enfermedades del tejido conectivo, causas misceláneas y aquellas sin diagnóstico. ${ }^{3}$

Tomando en cuenta lo expuesto, el caso corresponde a FOD clásica, en un adulto joven, y siendo las causas infecciosas las más frecuentes, los esfuerzos se dirigieron en ese sentido, aunque paralelamente se descartaron las otras causas. En pacientes menores de 65 años, las infecciosas más frecuentes son abscesos, endocarditis bacteriana subaguda, víricas y TB., ${ }^{4,5}$ Cabe señalar que la TB, en todas sus formas, es la causa infecciosa más frecuente, hasta un $40 \%$ de los casos según estudios españoles. ${ }^{4}$ En este caso, es evidente que el principal reto clínico consistió en diagnosticar o descartar una TB, particularmente extrapulmonar.

Es preciso analizar la PPD en este paciente, la cual fue de $10 \mathrm{~mm}$ de induración. En países desarrollados es positiva con este nivel si el paciente tiene factores de riesgo, como ser inmigrante de un país con alta prevalencia de TB, ser drogadicto intravenoso, residente o empleado de lugares de riesgo (por ejemplo hospitales) y niño menor de 4 años. ${ }^{6} \mathrm{El}$ paciente es nicaragüense, de un estrato socioeconómico bajo, de modo que se ubica en la población en riesgo, por lo que la prueba se interpretó como positiva. El $90 \%$ de aquellos
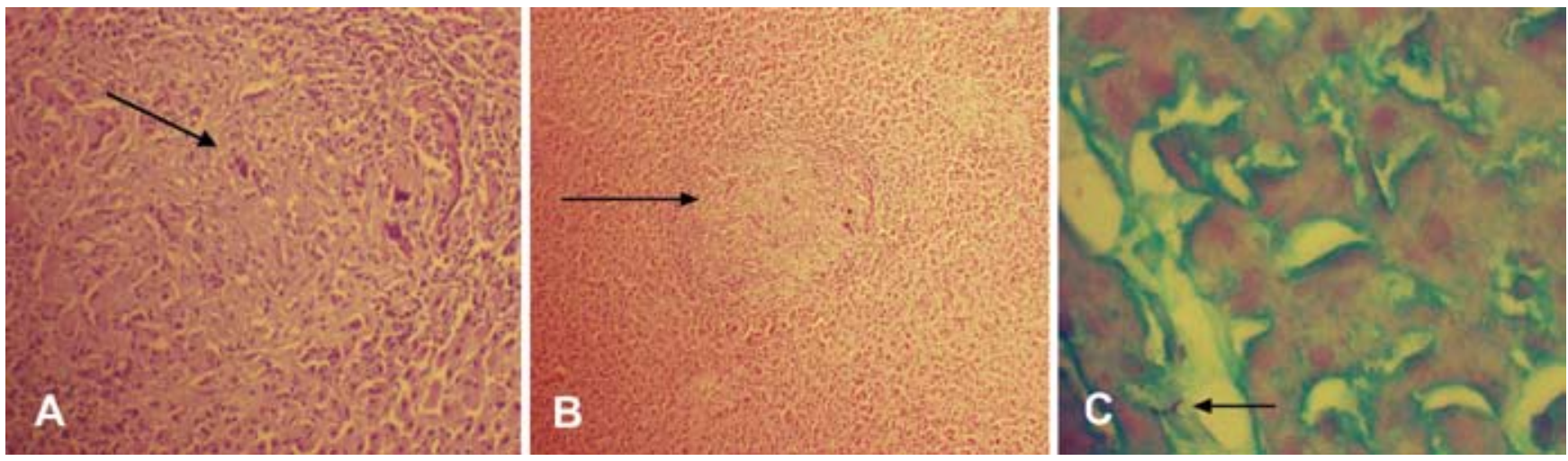

Figura 1. A, granulomas caseificantes; B, células gigantes rodeando un centro necrótico; C, tinción para bacilos alcohol-ácido resistentes con formas sugestivas, pero no diagnósticas. 
con PPD de $10 \mathrm{~mm}$ de induración tienen una infección por Mycobacterium tuberculosis. ${ }^{1}$

Por otro lado, aunque la mayoría de las series publicadas desacreditan la laparotomía exploradora como un método diagnóstico efectivo en FOD, debido a un bajo porcentaje de éxito $(<50 \%)$, en muchos casos la biopsia hepática "a ciegas" evidencia el diagnóstico final. Si bien es cierto que la TAC ha sustituido en parte a la laparotomía, ${ }^{7}$ dejando a esta última para los casos con anormalidades anatómicas evidentes radiológicamente. En este caso la tomografía no mostró alteraciones, por lo que era menos probable que la laparotomía fuera beneficiosa.

Cuando los estudios iniciales no brindan una orientación diagnóstica, quedan dos posibilidades. Si el estado general es bueno y no hay pérdida de peso importante, se recomienda dar de alta y reevaluar en 3 y 6 meses. Si el estado general es malo o existe deterioro progresivo, se debe evaluar la realización de una laparotomía (Figura 2), sobre todo si cursa con síntomas abdominales persistentes, masa abdominal palpable, ascitis de causa no determinada, estudios radiológicos abdominales anormales, hepatoesplenomegalia, derrame pleural, aumento de fosfatasa alcalina o pérdida de peso mayor del $10 \%, 4,8$ todas características ausentes en este caso.

Hasta un $10 \%$ de los casos persisten sin diagnóstico etiológico, es decir, son verdaderas FOD, generalmente con buena evolución y resolución espontánea de la fiebre, y solo un $10 \%$ de ellos tendrá un diagnóstico en los siguientes 5 años, con una mortalidad de apenas el 3\%. ${ }^{4}$ En casos sin diagnóstico definitivo que muestran deterioro progresivo, a menudo se utilizan pruebas terapéuticas, por ejemplo, antituberculosos, si se sospecha la infección por BK pero no se demuestra; ${ }^{5}$ antibióticos, si la sospecha es endocarditis o esteroides si se piensa en enfermedad de Still, ${ }^{2}$ pero tal proceder obedece más a la frustración del médico tratante que a la práctica médica basada en evidencia, por lo que se debe evitar.

En el paciente se documentó probable TB solo en el hígado, a pesar de tener ganglios paraaórticos aumentados de tamaño, estos fueron negativos por TB o granulomas; asimismo, se descartó una TB pulmonar por la clínica del paciente, la exploración mediante broncoscopía y las muestras microbiológicas que fueron negativas; por último, el más escéptico podría requerir de un estudio de médula ósea para descartar una forma diseminada de TB, pero un hemograma solo con plaquetopenia aleja esta posibilidad y, además, la evidencia dice que este examen es poco útil en el estudio de FOD en pacientes inmunocompetentes. ${ }^{2}$ Por estas razones, se considera que se trata de un raro caso de TB hepática primaria. Los casos descritos en la bibliografía tienen en común la alteración en las pruebas de función hepática, indicando necrosis hepatocelular o colestasis; en este caso las pruebas hepáticas estaban inalteradas. Por otra parte, la presencia de fiebre es más sugestiva de formas diseminadas, como la TB

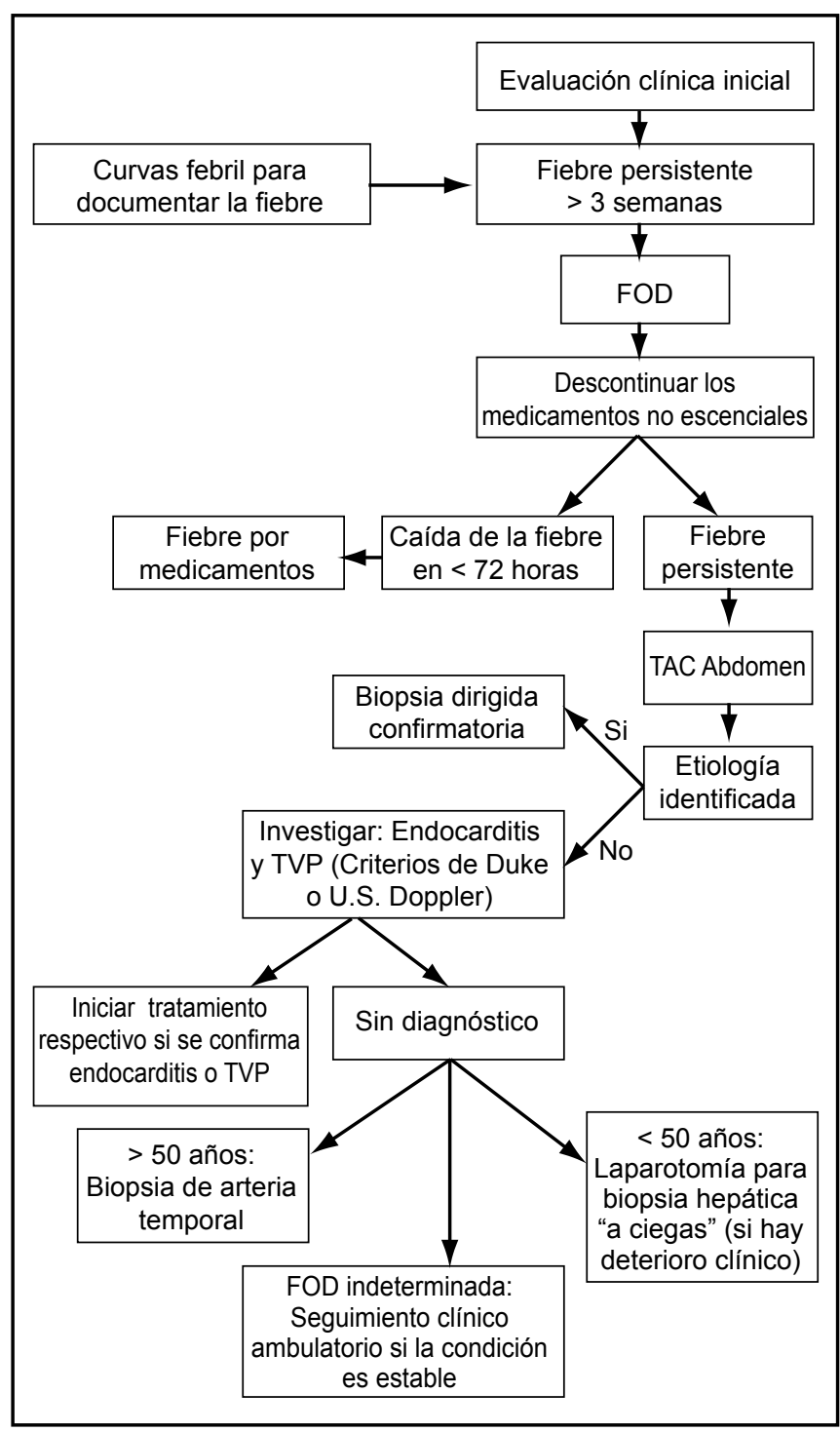

Figura 2. Algoritmo diagnóstico para FOD. TAC, tomografía axial computadorizada; TVP, trombosis venosa profunda. Basado en referencia 2.

miliar, la mayoría de las veces desde un foco pulmonar inadvertido, ${ }^{9}$ pero ausente en este caso.

Existe poca información sobre la TB hepática primaria, fundamentalmente debido a lo infrecuente de su aparición. La mayoría de los casos donde la TB afecta el hígado, lo hace en el marco de una infección sistémica diseminada ${ }^{9} \mathrm{o}$ se acompaña de una afectación intra-abdominal mayor, incluyendo bazo, peritoneo y ganglios. ${ }^{10}$ En el caso de una TB hepática primaria, el bacilo de Koch probablemente accede al hígado a través de una diseminación hematógena o linfática, inocua para los otros tejidos por razones desconocidas, o bien lo hace por diseminación biliar desde un foco intestinal. En los casos como este, donde hay granulomas hepáticos caseosos y formas sugestivas de bacilos tuberculosos con la tinción de Ziehl-Neelsen, donde se descarta definitivamente TB pulmonar, y el resto de la exploración abdominal es negativa por micobacterias y granulomas caseosos, el diagnóstico de TB hepática es el 
más apropiado, ${ }^{10} \mathrm{y}$ probablemente se trate de una reactivación de un foco presxistente.

Otro diagnóstico diferencial pertinente es el de los granulomas hepáticos; en este sentido cabe señalar que la TB es prácticamente la única entidad que forma necrosis caseosa en el hígado, ${ }^{11}$ pero no la única en producir granulomas. Otras enfermedades granulomatosas con asiento en el hígado son la sarcoidosis, la brucelosis, la beriliosis, la sífilis, la lepra, micosis como la histoplasmosis, la mononucleosis, el sida, la cirrosis biliar primaria, la hepatitis $\mathrm{C}$, la hepatitis autoinmune, las reacciones por drogas, los linfomas y vasculitis, como la granulomatosis de Wegener. ${ }^{11-13}$ Estas entidades producen granulomas epitelioides no caseosos, y generalmente afectan otros órganos, como en el caso de la sarcoidosis, donde la afectación de los ganglios pulmonares hiliares es casi invariable o la granulomatosis de Wegener con compromiso renal y de la vía aérea. En la mayoría de las series donde se reportan biopsias hepáticas, ${ }^{12}$ estas se efectúan como parte del estudio de un paciente con función hepática anormal, y no se incluye a pacientes con FOD es por esto que conocer la verdadera utilidad de la biopsia hepática "a ciegas" en FOD es difícil.

Formas atípicas de TB, como la hepática, han aparecido recientemente en pacientes inmunocomprometidos, como los pacientes con sida y los trasplantados. En este último grupo llama la atención la elevada incidencia de TB hepática en receptores de trasplante hepático, hasta del $48 \% .{ }^{14}$ Estas últimas consideraciones deben obligar a descartar inmunodeficiencias primarias o secundarias en los pacientes que muestran una infección inusual en frecuencia $y$ localización, como la TB hepática primaria.

\section{Abstract}

We present herein the case of a 29 a year old patient with fever, night sweats and weight loss. At the beginning he was thought to be suffering from classic dengue. Since the situation persisted for 3 weeks he was treated as classic fever of unknown origin. Due to non conclusive laboratory tests he underwent an exploratory laparotomy in order to get histologic samples, these showed caseous granulomas. This finding leads us to the diagnosis y hepatic tuberculosis. The patient was treated and the fever disappeared and 2 months later he started to gain weight.

\section{Agradecimiento}

A la Doctora Tilcia Y. López Q., médico asistente de Patología del Hospital Dr. Rafael Ángel Calderón Guardia, por su preciada y desinteresada colaboración con las fotografías de las biopsias del caso.

\section{Referencias}

1. Fitzgerald D, Hass DW. Mycobacterium tuberculosis. In: Mandell G, Benett's principles and pcantice of Infectous Diseases. Sixth Ed. Philadelphia, E Churchill. Livingstone; 2005; 2852-86.

2. Mourad O, Palda V, Detshy A. A Comprehensive Evidence-Based Approach to Fever of Unknown Origin. Arch Intern Med. 2003; 163: 545-51.

3. Burke A. Fever. Infec Dis Clin Nor Am. 1996, 10: 111-29.

4. Loja D, Vilca M. Granulmatous tuberculous hepatitis as cause of fever of unknown origin. Rev Gastroenterol Perú. 2002; 22: 324-9.

5. Chin C. Mycobacteriosis in patients with fever of unknown origin. Microbiol Immunol Infect. 2003; 36: 248-53.

6. Schluger N. Tuberculosis. Clin in Chest Med. 2005, 26: 247-71.

7. Ozaras R. Is laparotomy necessary in the diagnosis of fever of unknown origin? Acta Chir Belg. 2005; 105: 89-92.

8. D'Cruz K. Laparoscopic intervention in resistent hepatosplenic tuberculosis presenting as pyrexia of unknown origin. Trop Gastroenterol. 2005; 26: 40-2.

9. Surendra K, Alladi M, Anju S, Dipendra K. Milliary tuberculosis: new insights into an old disease. Lancet Inf Dis. 2005; 5: 415- 30.

10. Desai CS, Josh AG, Abraham P, Desai DC, Deshponde RB, Bhaduri A, Shah SR. Hepatic tuberculosis in absence of disseminated abdominal tuberculosis. Ann Hepatol. 2006, 5: 41-3.

11. Geraint J. A clinicopathological classification of granulomatous disorders. Postgrad Med J. 2000; 76:457-465.

12. Gaya D, Thorbum D, Oien KA, Morris AJ, Stanley AJ. Hepatic granulomas: a 10 year single centre experience. J Clin Pathol. 2003; 56:850-53.

13. Matheus T, Muñoz S. Granulomatous liver disease and cholestasis. Clin Liver Dis. 2004; 8:229-246.

14. Singh N, Paterson D. Mycobacterium tuberculosis Infection in SolidOrgan Transplant Recipients: Impact and Implications for Management. Clin Inf Dis. 1998; 27:1266-77.

Servicio de Medicina Interna. Hospital Dr. Rafael Ángel Calderón Guardia, Caja Costarricense de Seguro Social

Abreviaturas: FOD, fiebre de origen desconocido; HCG, Hospital Rafael Ángel Calderón Guardia; PPD, derivado proteico purificado, TB, tuberculosis.

Correspondencia: Manuel Antonio Villalobos Zúñiga. Hospital Calderón Guardia.E-mail: manuel70I@costarricense.cr.

Apartado postal 2434-1000 San José. 